\title{
SPECTRAL PARTITIONING AND SWELLS IN THE BLACK SEA
}

\author{
Gerbrant Ph. Van Vledder ${ }^{12}$ and Adem Akpinar ${ }^{3}$
}

\begin{abstract}
The swell climate of the Black Sea has been determined using a long-term 31-year wave hindcast with the thirdgeneration spectral wave model SWAN in combination with spectral partitioning. This technique enables decomposing wave spectra into individual wave systems representing wind seas or swells and computing integral wave parameters of each partition. Results are presented of the partition technique and of spatial and seasonal characteristics of wind sea and swell systems. In addition, the average amount of swell energy and the occurrence probability of dangerous crossing sea states are determined.
\end{abstract}

Keywords: SWAN, Wind-wave modelling, Swell climate, Spectral Partitioning, Black Sea

\section{INTRODUCTION}

Low-frequency wind generated swell waves have a special place in offshore and coastal engineering as they occur independent of the local wind. These waves can be particularly dangerous as they may have relatively large periods in combination with small heights in contrast to storm waves where heights and periods are strongly coupled due to the physical effects of non-linear four-wave interactions (cf. Hasselmann et al. (1973) and Young and Van Vledder (1993). Therefore, information on their occurrence probabilities is important to estimate down-time of ports, the optimal design of wave energy convertors and ship motions in cross seas. Although the Black Sea is limited in size, it is still large enough to generate swell waves in storm systems, which then radiate to other sides of this sea. To properly cope with these waves, reliable information on their statistical properties is important for many offshore and coastal engineering applications, both in terms of their heights as well as their periods.

The first major study investigating the swell climate in the Black Sea was performed by Berkün (2007). He analyzed wave model output of the ECMWF WAM model implementation for the Black Sea. This study, however, was limited to analyzing wave parameters of significant wave height, peak period and mean wave direction for the wind sea and one swell component at 10 locations along an eastwest transect in the Black Sea covering a period of 65 months (about 5.5 years). The ECWMF model applied, used a wind based criterion to split a modelled frequency-direction wave spectrum in a wind sea part and a swell part. The wind sea part of this spectrum was defined as that part of the wave spectrum lying in the influence zone of the wind using a wave age criterion:

$$
c_{p} \leq 1.2 \times 28 u_{*} \cos \left(\theta_{w}-\theta\right)
$$

in which $c_{\mathrm{p}}$ is the phase velocity of a wave component, $u_{*}$ is the friction velocity, $\theta_{\mathrm{w}}$ is the wind direction and $\theta$ is the direction of a wave component. This equation traces out a parabolic boundary in the frequency-direction space of a wave spectrum.

Spectral shapes were analyzed by Yilmaz (2007) using buoy data collected intermittently over 101 months in the period from 1994-2003 at the buoy locations of Sinop, Gelendzhik and Hopa. Of particular interest are her estimates of percentages of sufficiently well-resolved multi-peaked frequency spectra, each having a minimum total significant wave height of $0.5 \mathrm{~m}$. A limitation of her study was that only frequency spectra were considered, and that no distinction was made between wind seas and swell waves. These initial works of Berkün (2007) and Yilmaz (2007) were a good step towards a more elaborate statistical description of the swell climate.

In this study these analyses were extended by determining the swell climate in the entire Black Sea, by using a longer simulation period and by using a more advanced method to partition the wave spectrum into a wind sea partition and one or more swell partitions. For the partitioning the spectral partitioning technique recently implemented in the SWAN wave model was applied. This approach enabled a more detailed splitting of a wave spectrum into its constituting components, not limited to one swell partition. Another advantage is that our analysis is not confined to a limited number of output

\footnotetext{
${ }^{1}$ Delft University of Technology, Civil Engineering and Geosciences, The Netherlands

${ }^{2}$ Van Vledder Consulting, Olst, The Netherlands

${ }^{3}$ Uludag University Department of Civil Engineering, Gorukle Campus, Bursa, 16059, Turkey 
locations, but also that the spatial distribution of the swell climate in the Black Sea and Sea of Azov can be determined. This facilitated examining patterns of swell propagation over the entire Black Sea.

\section{SPECTRAL PARTITIONING}

A new feature of SWAN, first added to version 41.10, is spectral partitioning as part of internal post-processing. This technique identifies areas in the frequency-direction spectrum each associated to a local spectral peak. The splitting technique is based on the inverse watershed algorithm as described in Hanson and Phillips (2001) and Portilla et al. (2009). The first step in this method is to find the spectral bins in frequency-direction space that are linked to a local peak by following a line of steepest ascent. The second step is to find the spectral peak(s) associated to the local wind, while all other possible peaks are considered to belong to one or more swell partitions. The criterion to assign one or more peaks as wind seas is described in Hanson and Phillips (2001) and contrary to Eq. (1), formulated in terms of wind speed $U_{10}$ :

$$
c_{p} \leq C_{\text {wind }} \times U_{10} \cos \left(\theta_{w}-\theta\right)
$$

where $U_{10}$ is the wind speed at a height of $10 \mathrm{~m}$. Hanson and Phillips (2001) argue that the relative large factor $C_{\text {wind }}=1.5$ is chosen to ensure that all possible wind sea peaks are included. In case of a single peaked spectrum the wave-age criterion will assign it to be either a wind sea peak of a swell peak. An interesting application of this splitting technique in identifying climatology of families of wave systems can be found in Portilla et al. (2015). In SWAN a more generous value of $C_{\text {wind }}=1.7$ is used.

Figure 1 shows an example of the partitioning of a 2D-frequency-direction wave spectrum. Each panel shows a polar plot of the normalized wave spectrum. The solid line indicates the area of influence of wind according to Eq. (2) with $C_{\text {wind }}=1.7$. As can be seen, this line intersects different partitions. In contrast to the ECWMF procedure outlined above, all whole partitions whose peaks lie in the area of influence are considered as the wind sea partition. All other significant partitions are considered as swell partitions, even if parts of them are under the influence of wind. Hereafter, for each partition a number of spectral parameters are computed including a parameter WFRAC indicating the relative area of a 2Dwave spectrum lying within the influence of the wind according to Eq. (2). Usually, this parameter is the greatest for the wind sea partition.
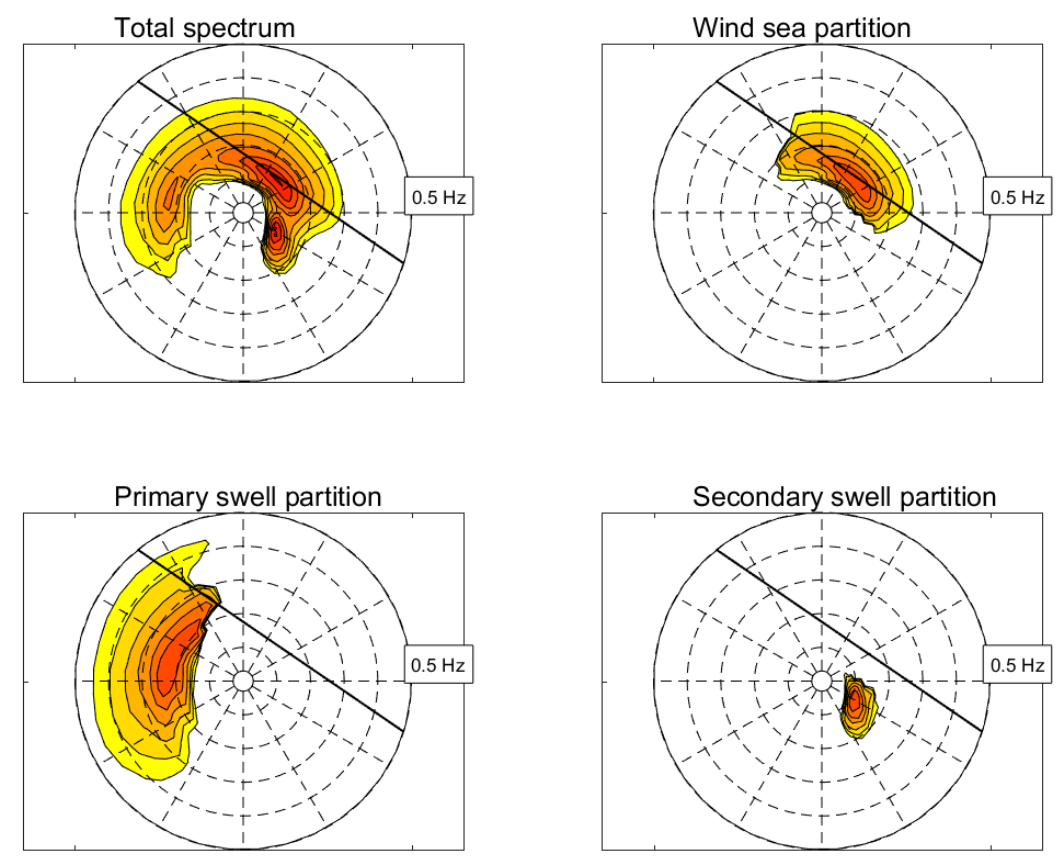

Figure 1. Normalized polar spectra of full spectrum (upper left panel), wind sea partition (upper right panel), primary swell partition (lower left panel) and secondary partition (lower right panel) for 27 Oct, 1996, 12:00 hours at $\lambda=40^{\circ}$ and $\varphi=43^{\circ}$. The black line indicates the wave-age criterion Eq. (2). 
It is noted that the basic routines for the partitioning of wave spectra and the computation of their properties are the same as those implemented in the WaveWatch III model. This shared feature is beneficial when processing output from both models. Spectral partitioning in SWAN is activated with sub-commands starting with the letter combination PT in the main commands for generating Table and Block output. An example of SWAN input lines for generating output (here non-stationary) of all possible integral parameters of each partition is as follows:

\section{TABLE 'swell' HEADER 'table\BS_PARTIT_201702.tab' TSEC Time XP YP \& PTHSIG PTRTP PTWFRAC PTDIR PTDSPR PTWLEN PTSTEEP \& OUTPUT 19960201:000000 1.0 hr}

\section{BLOCK 'COMPGRID' NOHEAD 'blocklbs_201702.mat' LAY 3 XP YP \& PTHSIG PTRTP PTWFRAC PTDIR PTDSPR PTWLEN PTSTEEP \& OUTPUT 19960201:000000 $3 \mathrm{hr}$}

\section{BLOCK 'COMPGRID' NOHEAD 'blocklbs_par_201702.RAW' LAY 3 XP YP PARTIT \& OUTPUT 19960201:000000 $3 \mathrm{hr}$}

in which PTHSIG, PTRTP, PTWFRAC, PTDIR, PTDSPR, PTWLEN and PTSTEEP represent for each partition the significant wave height, relative peak period, fraction of spectral area actively driven by wind, mean wave direction, directional spreading, mean wave length and mean wave steepness. Partitions of which their peak value is not in area of influence of the wind, are treated as a swell partition, even if an area of this partition, as expressed by the value of the parameter PTWRAC, is under the influence of the wind.

By default, results of 10 partitions are output, even if a wave spectrum has only one partition. In case less than 10 partitions are identified, the parameter values for non-existing peaks are filled with zeros. The first numbered partition PT01 is always the wind sea one, even for the case that no wind sea is present. The swell partitions PT02 to PT10 are sorted with decreasing significant wave height.

The third command containing the keywords 'RAW' and PARTIT generates a so-called 'raw' table with information on each non-empty partition for all grid points and time steps containing parameter values of wind, significant wave height, peak period, mean wave direction and directional spreading. This output was defined to enable tracking in space and time of evolving wave systems (see the Wavewatch manual, 2009, and Devaliere et al., 2007). Details on the file format and meaning of different parameters can be found in http://polar.ncep.noaa.gov/waves/workshop/pdfs/

WW3-workshop-exercises-day4-wavetracking.pdf.

Spectral partitioning may increase run time depending on the extent of output requests. It is noted that no output is generated of the partitioning itself, e.g. in the form of a 2D-matrix filled with integers indicating the partition number of each spectral frequency-direction bin. Further details of using spectral partitioning can be found in the SWAN manual (SWAN team, 2016).

\section{MODEL SETUP}

To study the swell climate in the Black Sea the SWAN model (Booij et al., 1999) was applied in non-stationary mode to simulate 31 years starting on Jan. 1, 1979 till Dec. 31, 2009. In our nonstationary simulations we applied the same model set-up as used in Van Vledder and Akpinar (2015). This included a spatial grid 225 by 120 grid cells in the longitude range $27^{\circ} \mathrm{E}-42^{\circ} \mathrm{E}$ and latitude range $40^{\circ} \mathrm{N}$ to $48^{\circ} \mathrm{N}$, with a step size of $\Delta \lambda=1 / 15^{\circ}$ and $\Delta \varphi=1 / 15^{\circ}$. The spectral resolution consisted of 36 directions equally spaced with a $10^{\circ}$ step and 43 frequencies logarithmically distributed in the range 0.03 $\mathrm{Hz}-1.5 \mathrm{~Hz}$, such that each frequency is $10 \%$ higher than the previous frequency. The relatively high upper frequency was chosen to improve accuracy for low wind speeds.

The time step of the non-stationary computations was 15 minutes with one 1 iteration per time step as recommended by Akpinar et al. (2012). Following Akpinar et al. (2012) and Van Vledder and Akpinar (2015), hourly CFSR wind fields were used to drive the SWAN wave model.

The physical settings are based on the default settings of the third-generation wave physics according to Komen et al. (1984, 1994). Linear wave growth is based on Cavaleri and MalanotteRizzoli (1981), and exponential wind input is based on Snyder et al. (1981) as rescaled by Komen et al. (1984). Whitecapping dissipation is based on Komen et al. (1994) using DELTA=1 as recommended by 
Rogers et al. (2003). The non-linear interactions are estimated using the Discrete Interaction Approximation (DIA) of Hasselmann et al. (1985) using $\lambda=0.25$ and $C_{\mathrm{n} 14}=3 \times 10^{7}$. Bottom friction is according to the JONSWAP formulation of Hasselmann et al. (1973) with $C_{\mathrm{f}, \mathrm{JON}}=0.038 \mathrm{~m}^{2} \mathrm{~s}^{-3}$. Triad interactions were not activated.

Table output was generated every hour at 214 locations regularly spaced at a grid of $0.5^{\circ}$ by $0.5^{\circ}$, whereas block output at all computational points was generated every 3 hours. For illustration purposes three output points were selected, one in the western part, the middle part and the eastern part of the Black Sea. The positions of all output locations and the selected output points are shown in Figure 2.

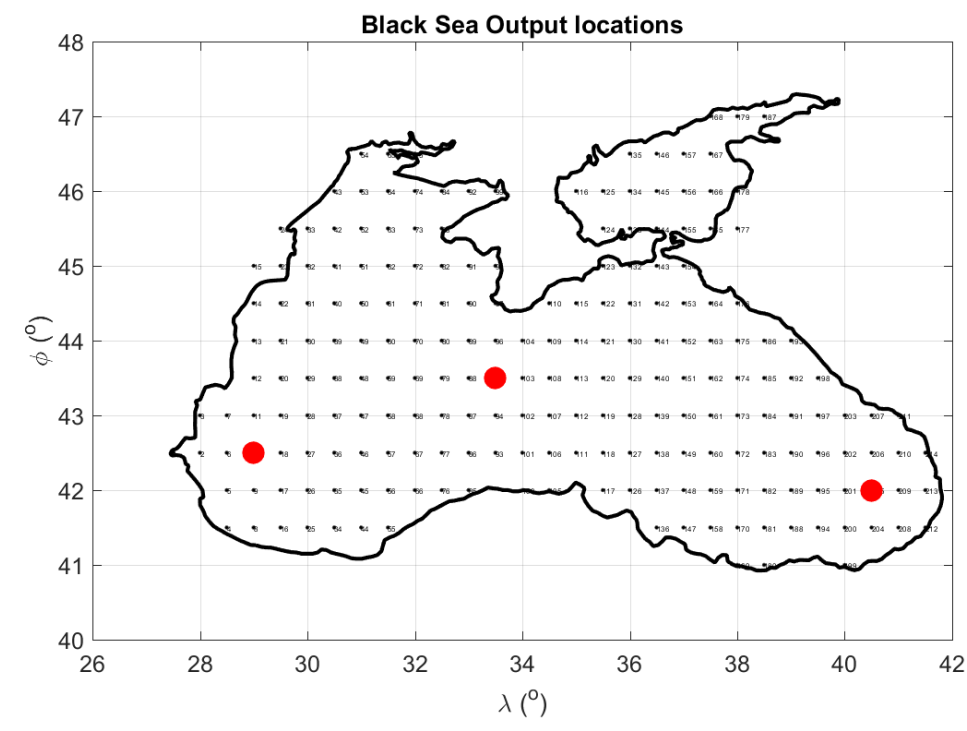

Figure 2. Outline of the Black Sea and positions of selected output locations (red dots) circles.

\section{RESULTS}

An example of spectral partitioning is shown in Figure 3 in the form of time series of wind speed $U_{10}$ (upper panel), the total and partitioned significant wave heights of the wind partition and the primary and secondary swell partition (top second panel), associated peak periods (top third panel) and the corresponding directions, including wind (bottom panel).

As can be seen the total significant wave height (black line) is usually composed of only a wind sea (blue line) and where the significant wave height is strongly coupled with the local wind speed. Quite often a significant primary swell (red lines) occurs sometimes supplemented with secondary swell (green lines). The associated peak periods of the wind sea are strongly related to their significant wave height indicating actively driven wave field. As expected the swell periods usually exceed the wind sea peak period. The mean directions (lower panel) show the wind sea direction follows the wind directions with some expected delay (cf. Van Vledder and Holthuijsen, 1993). 

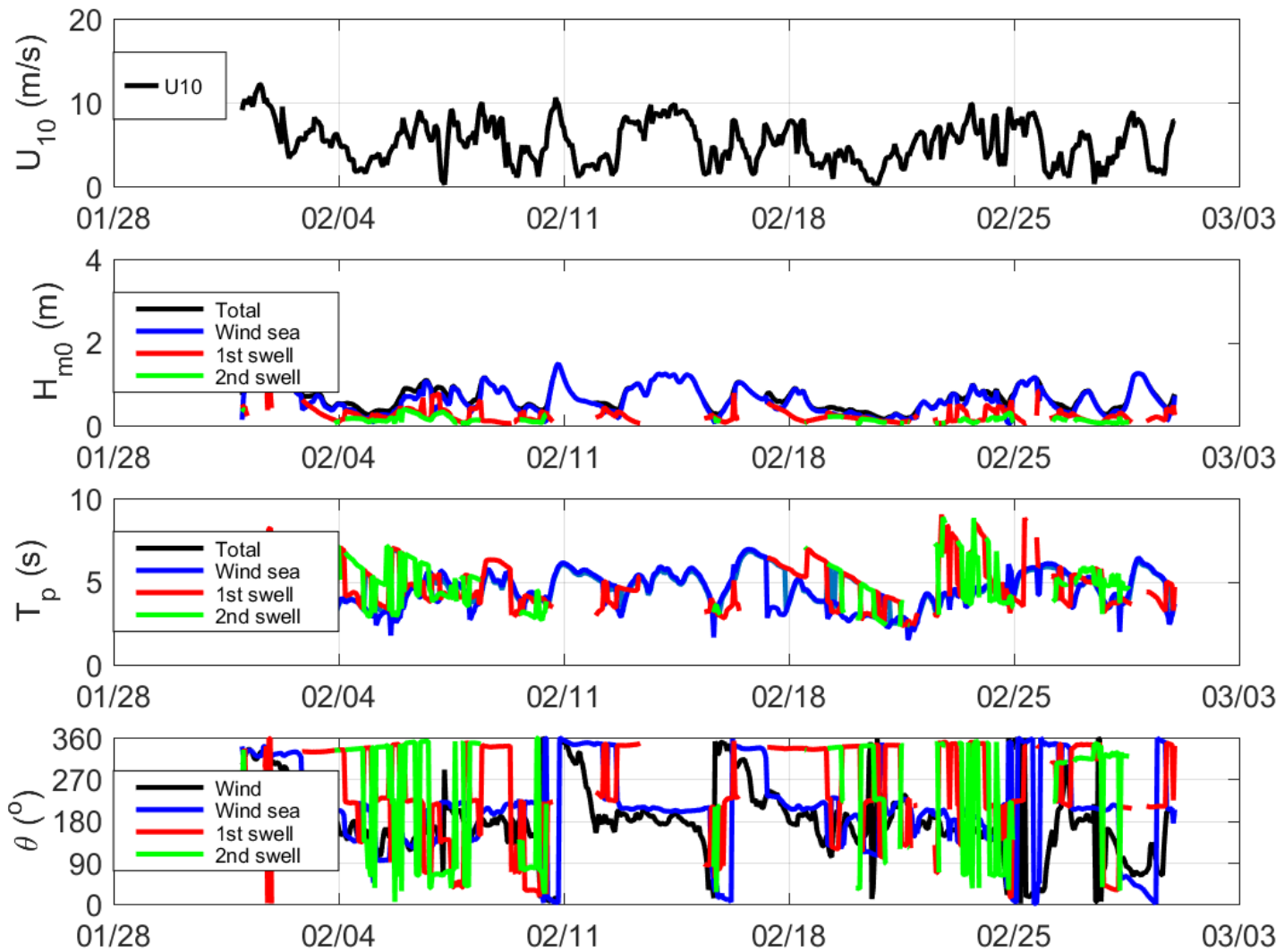

Figure 3. Time series of wind speed U10 (upper panel), significant wave heights (top second panel), peak periods (top third panel) and wind and mean wave directions (bottom panel) for output location P95 and the month of February in 1996.

An example of non-partitioned and partitioned wave fields is shown in the Figures 4 and 5 for the significant wave height $H_{\mathrm{m} 0}$ and peak period $T_{\mathrm{p}}$, respectively for 27 October, 1996, 12:00 hours. It is noted that this moment of time is associated to the storm event analyzed in Van Vledder and Akpinar (2015). The upper left panel in Figure 4 shows the spatial variation of the total significant wave height $\left(H_{\text {sig }}\right)$, the upper right panel shows the wind sea partition (HSPT01), the lower left panel shows the primary swell partition (HSPT02), and the lower right panel shows the spatial variation of the secondary swell partition (HSPT03). Figure 5 shows the associated peak periods for the same moment of time. The arrows in these two figures show the mean wave direction scaled with the related significant wave height.

The results in these figures clearly illustrate the power of spectral partitioning showing the existence of a strong wind sea storm system in the southeastern part of the Black Sea and a swell system moving to the south-west in the southwestern part of the Black Sea. In addition, a small secondary swell system exists in the wake of the storm system. The significant wave height peaks in the storm with heights up to 7 meters and peak periods up to 10 seconds. The swell heights are about $1.8 \mathrm{~m}$ with peak periods up to 10 seconds. A remarkable feature is that the peak periods of the western primary swell systems are highest along their northern regions (about $45^{\circ} \mathrm{N}$ ). The northeastern primary swell system is trailing behind the moving storm system. The origin of this swell system is due to the drop in the wind speed such that these waves are not any longer under the influence of the local wind, which by definition makes these waves swell.

The secondary swell system in the northeastern area near the Russian town of Gelendzhik is a small system having the same origin as the nearby primary swell system but having a spectral peak sufficiently separated from the wind sea and primary swell peaks. In case these peaks are close in spectral space, one may combine these peaks into one swell peak. In fact such merging of peaks is discussed in Hanson and Phillips (2001) and Portilla et al. (2009) to retain only significant peaks. 

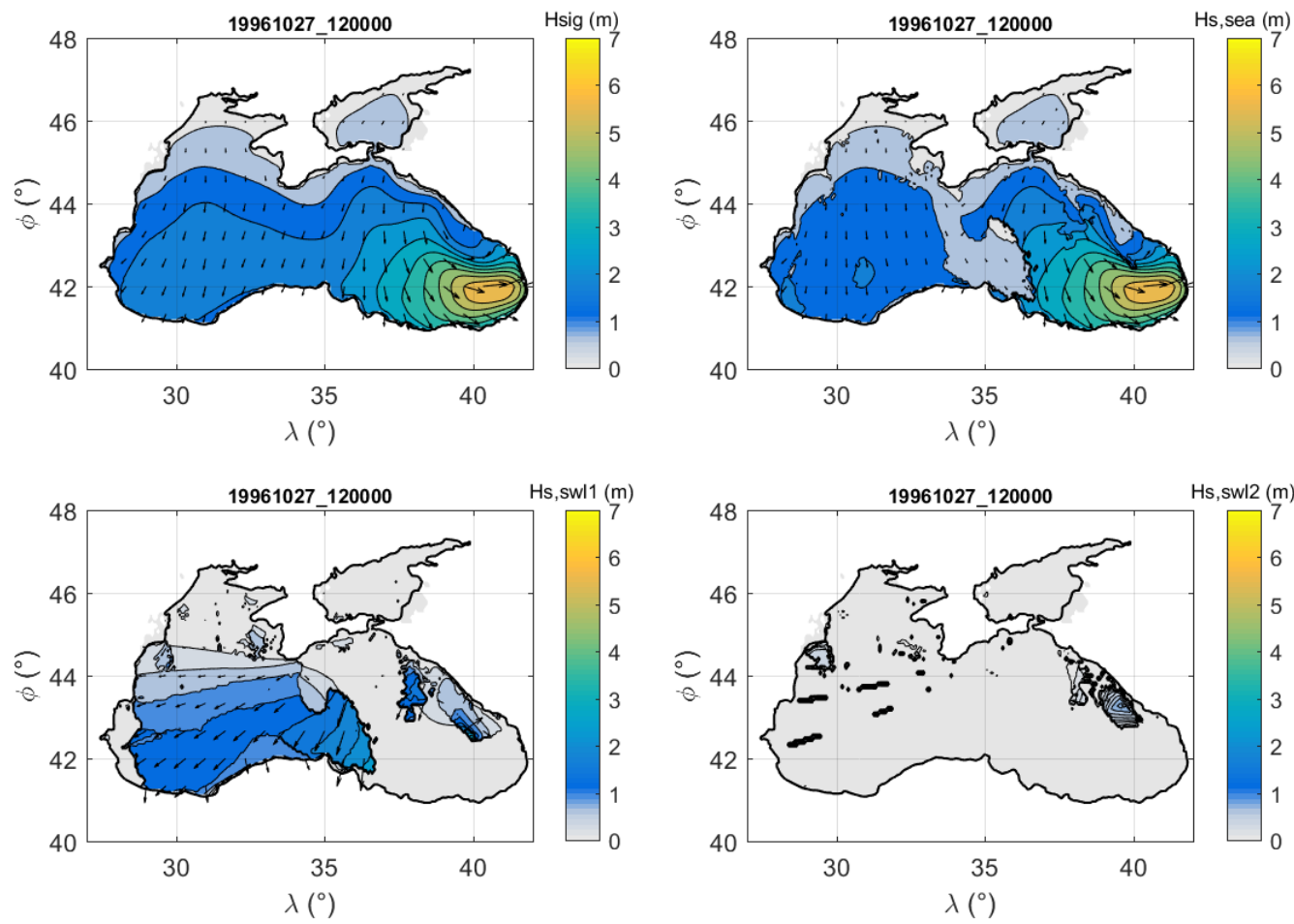

Figure 4. Spatial variation of the total significant wave height (upper left panel), wind sea partition (upper right panel), primary swell partition (lower left panel) and secondary swell partition (lower right panel) for 27 October, 1996, 12:00 hours. The arrows indicate the mean wave direction of each partition scaled with the associated significant wave height.
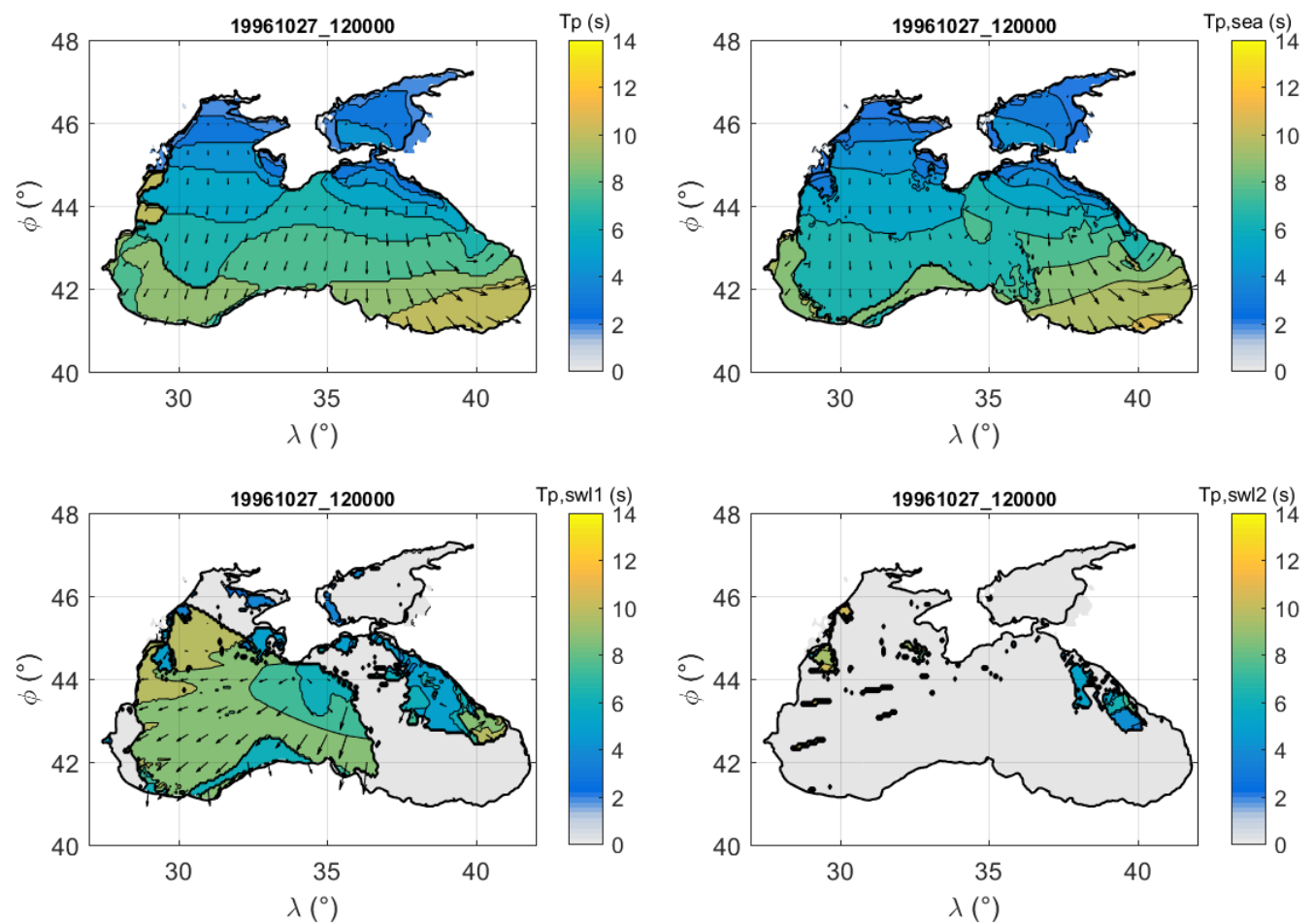

Figure 5. Spatial variation of the overall peak period (upper left panel), wind sea partition (upper right panel), primary swell partition (lower left panel) and secondary swell partition (lower right panel) for 27 October, 1996, 12:00 hours. The arrows indicate the mean wave direction of each partition scaled with the associated significant wave height. 


\section{CLIMATE RESULTS}

The previous figures showed the ability of spectral partitioning tool in decomposing the wave field in its constituting components. From an application point of view it is interesting to know how much wave energy in the Black Sea can be attributed to swell. This is illustrated in Figure 6 showing the average seasonal contribution of swell energy to the total wave energy computed as:

$$
S W_{\text {perc }}=\frac{\sum_{i} H_{s, \text { swell }, i}^{2}}{\sum_{i}\left(H_{s, \text { swell }, i}^{2}+H_{s, \text { sea }, i}^{2}\right)} \times 100 \%
$$

where $i$ loops over all time steps at a certain location.

In the present analysis seasons are defined as three-month periods with winter containing the months December, January and February; spring containing the months March, April and May; summer containing the months June, July and August and autumn containing the months September, October and November.

The results show that the Sea of Azov has the lowest amount of swell energy. This is not surprising as wave systems are generally smaller in spatial extent than the wind systems generating waves. In the Black Sea basin, the highest amount of swell energy occurs in the winter season just east of the middle of this sea. It is surprising that a high percentage of swell energy also occurs in the summer season along the northeastern Russian coast. For the spring season no particular area can be identified where the contribution of swell energy strongly deviates from the overall mean in the Black Sea. For the summer season it is found that the relatively swell rich areas occur in the western and eastern part of the Black Sea. The autumn season shows relatively large contributions of swell energy in the northwestern areas and also in the same area where much swell energy occurs in the winter season.
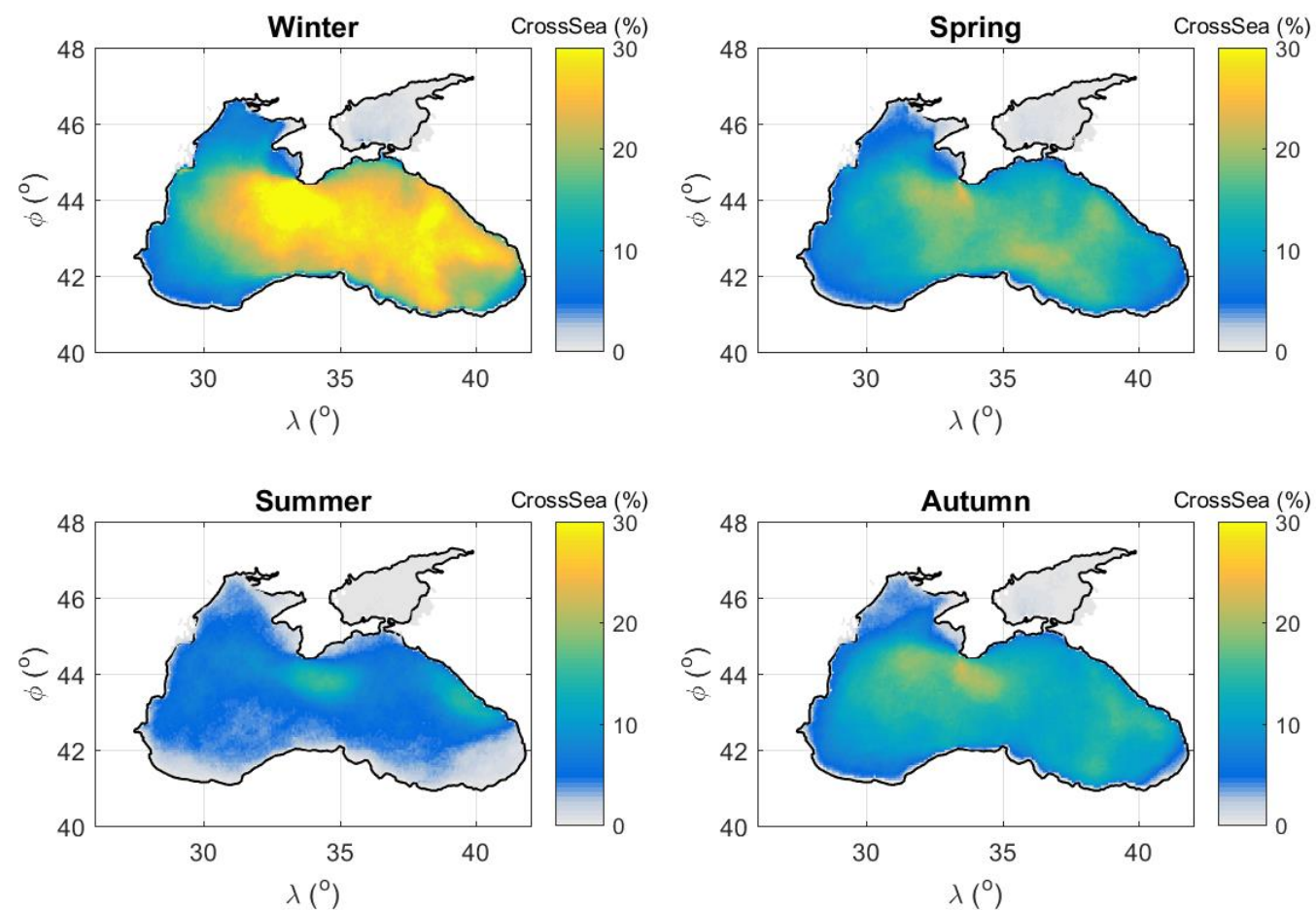

Figure 6. Spatial variation of percentage of swell energy in the Black Sea per season based on hindcast results from 1979-2009.

The next step in our analysis is to assess the maximum wind sea and swell conditions that occur in the Black Sea. To that end the maximum significant wave height $H_{\mathrm{m} 0}$ and peak period $T_{\mathrm{p}}$ that occurred in the Black Sea in the period 1979-2009 were determined for all grid points. The Figures 7 and 8 show the maximum significant wave heights for wind sea and swell, respectively. The subplots show the spatial distribution per season. The Figures 9 and 10 shows the associated distributions of peak periods. 
In line with previous findings (Akpinar et al., 2016) the highest wind sea conditions are found in the southwestern and southeastern part of the Black Sea with winter being the most severe season and summer the calmest. A similar pattern is found for the maximum swell height, but with a few marked differences. The spatial distribution is less smooth than the wind sea and it is spread over a wider area as propagate as swell waves over the Black Sea independent of the local wind. Concerning the spatial distribution of the maximum peak periods, less marked differences are found between wind seas and swell systems. Still, a patch of relatively high swell peak periods is found in autumn in the southeastern part of the Black Sea. This comparison shows that swells in the Black Sea are strongly coupled to the wind seas in which they originate. Two mechanisms play a role in the origin of swell waves. Firstly, as a storm system moves over the Black Sea it leaves an area with lower wind speeds in its 'wake', which is then considered as swell in our definition (Eq. 2). In addition, such moving systems commonly have turning winds often leading to a decoupling of the old wave system becoming swell and a newly developed wind sea (Van Vledder and Holthuijsen, 1993). Secondly, as swell waves travel faster than the local wind speeds, they escape the storm area. Both mechanisms lead to swell waves that may propagate over the entire Black Sea giving a local surfer or sailor a signal from a distant storm.

The simultaneous occurrence of wind seas and swell can be dangerous for ships crossing the Black Sea. The spectral partitioning method was used to determine the occurrence probability of multi-peaked wave systems. To arrive at statistical meaningful results a 'dangerous' sea state was defined as having at least a wind sea peak, at least one swell peak both having a significant wave height of at least $0.25 \mathrm{~m}$ and propagation directions deviating more than $90^{\circ}$. The result of this analysis is shown in Figure 11 for each season. The highest probability of occurrence of such multi-peaked wave systems occurs in winter the lowest in summer. The occurrence probabilities have lower values along the coast lines, which indicates that such sea states need larger fetches to develop. The Sea of Azov shows the least amount of multi-peaked wave spectra, indicating that it is limited in a physical sense to develop multi-peaked wave spectra. A comparison with results from Yilmaz (2007) is difficult to make due to differences in the method to detect multi-peaked spectra. Despite these differences an estimate is made using the percentage of multi-peaked spectra for location Hopa collected intermittently in 41 months in the years 1994-1999, using the criterion that the significant wave height of the wind sea peak (HSPT01) and primary swell peak (HSPT02) both exceed $0.25 \mathrm{~m}$ and that their directions differ at least $90^{\circ}$, yielding the percentages $10 \%, 8 \%, 5 \%$ and $5 \%$ for winter, spring, summer and autumn, respectively. These numbers are much smaller than those of Yilmaz (2007), viz. 27\%, 28\%, 25\% and 22\%, probably due the additional directional difference criterion and the intermittency of the observations.

A detailed look at the partitioned wave climate is by inspection of the wind and wave roses. Figures 12 to 14 show the wind and wave roses for the three output locations (L10, L95 and L205) as shown in Figure 2. The upper left rose is the local wind rose derived from the CFSR wind data. The upper right rose shows the wave rose of the total wave condition based on the overall significant wave height. The lower roses show the wind sea wave height and all swell wave heights (i.e. multiple swell heights higher than $0.05 \mathrm{~m}$ occurring at the same moment of time are included in the statistics). The roses show interesting relations between local wind, local wind sea and distantly generated swell waves.

For location L10 in the western part of the Black Sea strong similarities between the wind, total wave and wind sea roses occur, especially for the north-eastern sector. Differences in the distribution are due to upwind fetch-restrictions, e.g. for waves originating south of this location. The swell is mainly coming from easterly directions, indicating they were generated in the middle and even eastern part of the Black Sea.

For location L95, in the middle part of the Black Sea, the wind rose shows more or less an evenly distribution over the western, northern and easterly sectors. This is not reflected in the wave roses, which show a gap for northerly directions due to the proximity of land in the North restricting fetch lengths. The swell rose is dominated by strong contributions from the northwest and the east. It is also notable that the contribution of relatively higher swell waves is smaller than those of the wind sea components. This suggests that this part of the Black Sea is not a primary region for the generation of swell waves.

For location L205, in the eastern part of the Black Sea, the wind rose shows a strong bi-modal distribution, which is partly reflected in the total wave and wind sea roses. The swell rose strongly deviates from the wind rose, with a strong contribution from northwesterly directions and hardly any contribution from southerly and southeasterly directions. 

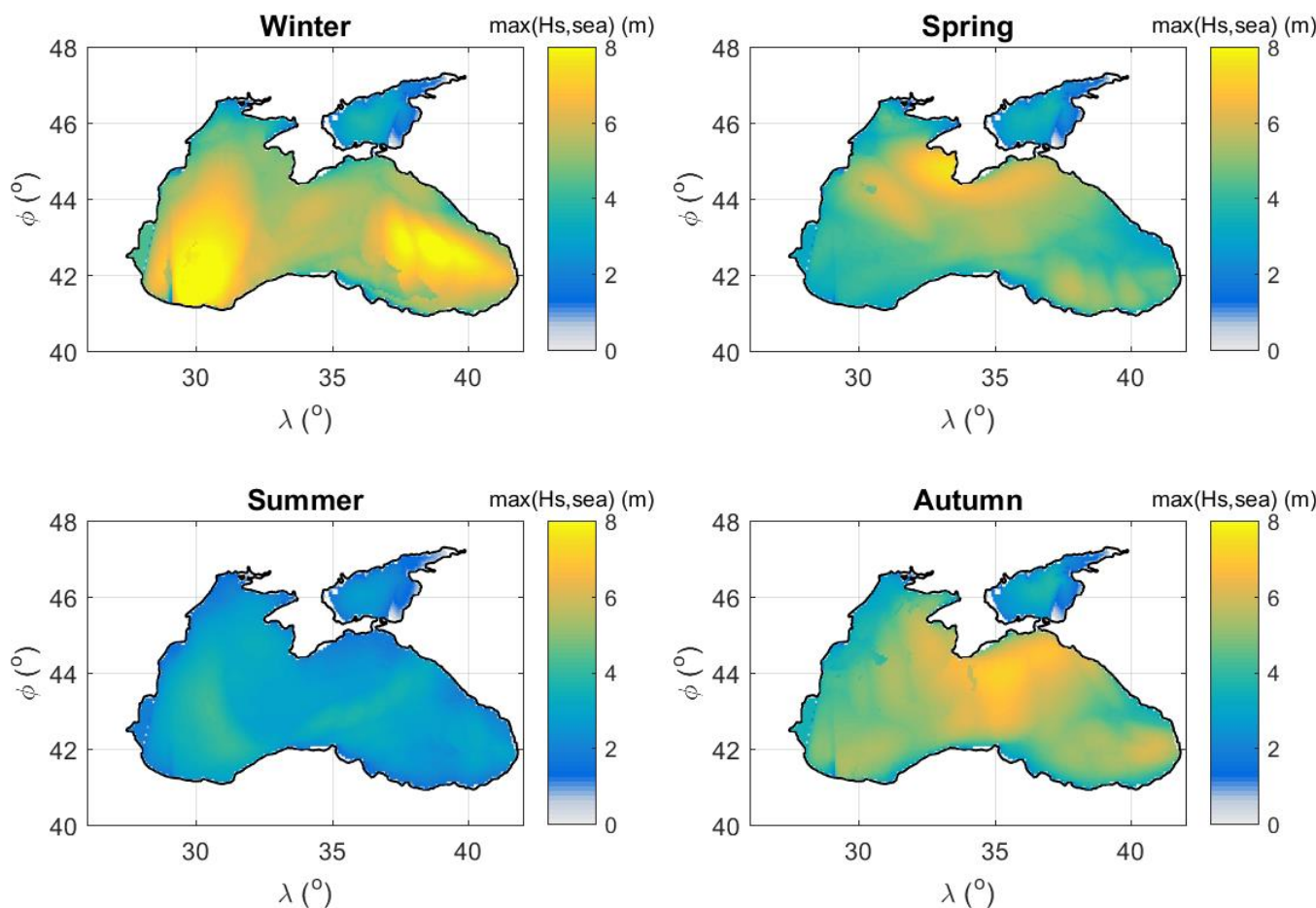

Figure 7. Spatial variation of the maximum significant wave height of wind seas per season based on hindcast results from 1979-2009.
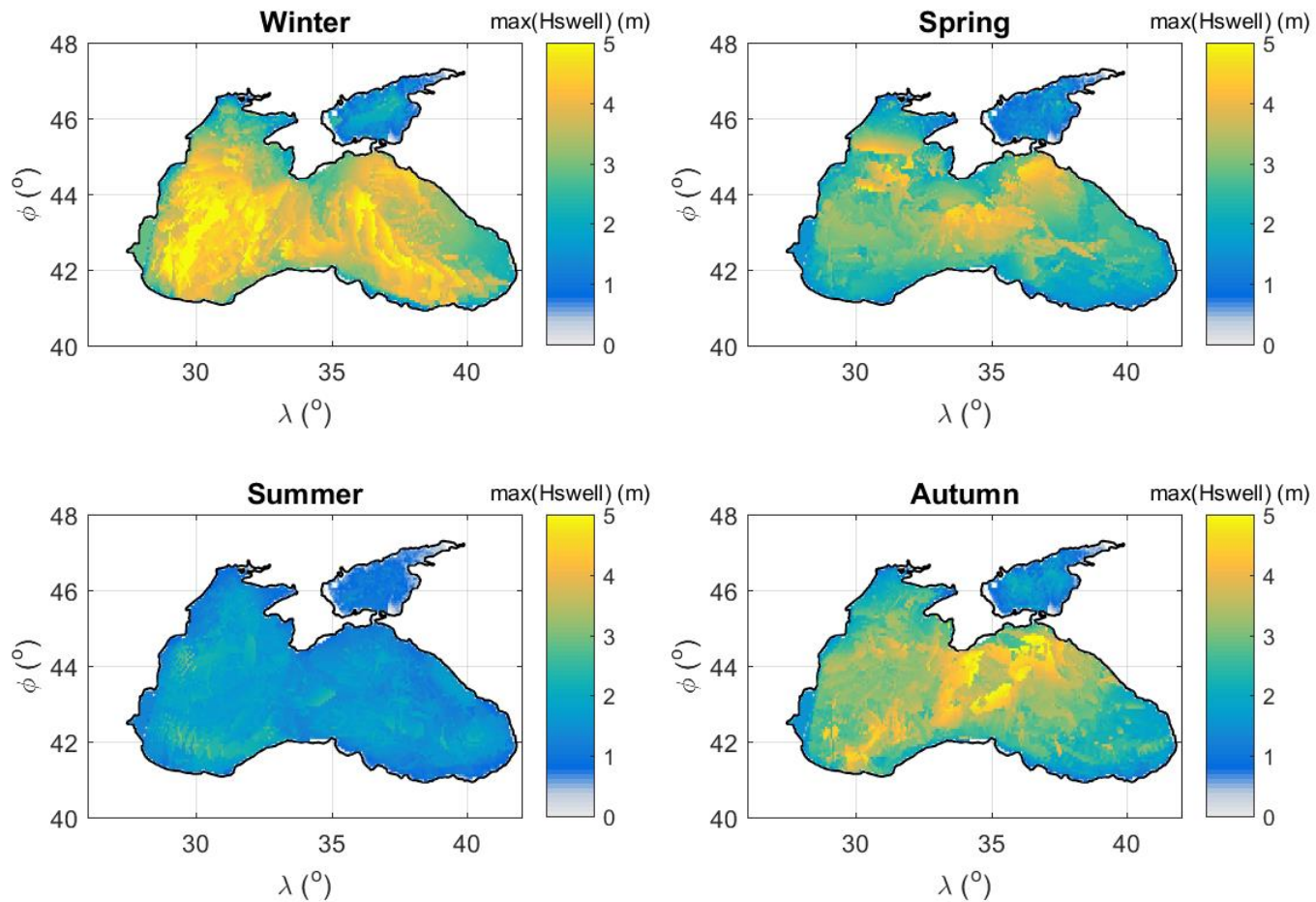

Figure 8. Spatial variation of the maximum significant wave height of swell waves per season based on hindcast results from 1979-2009. 

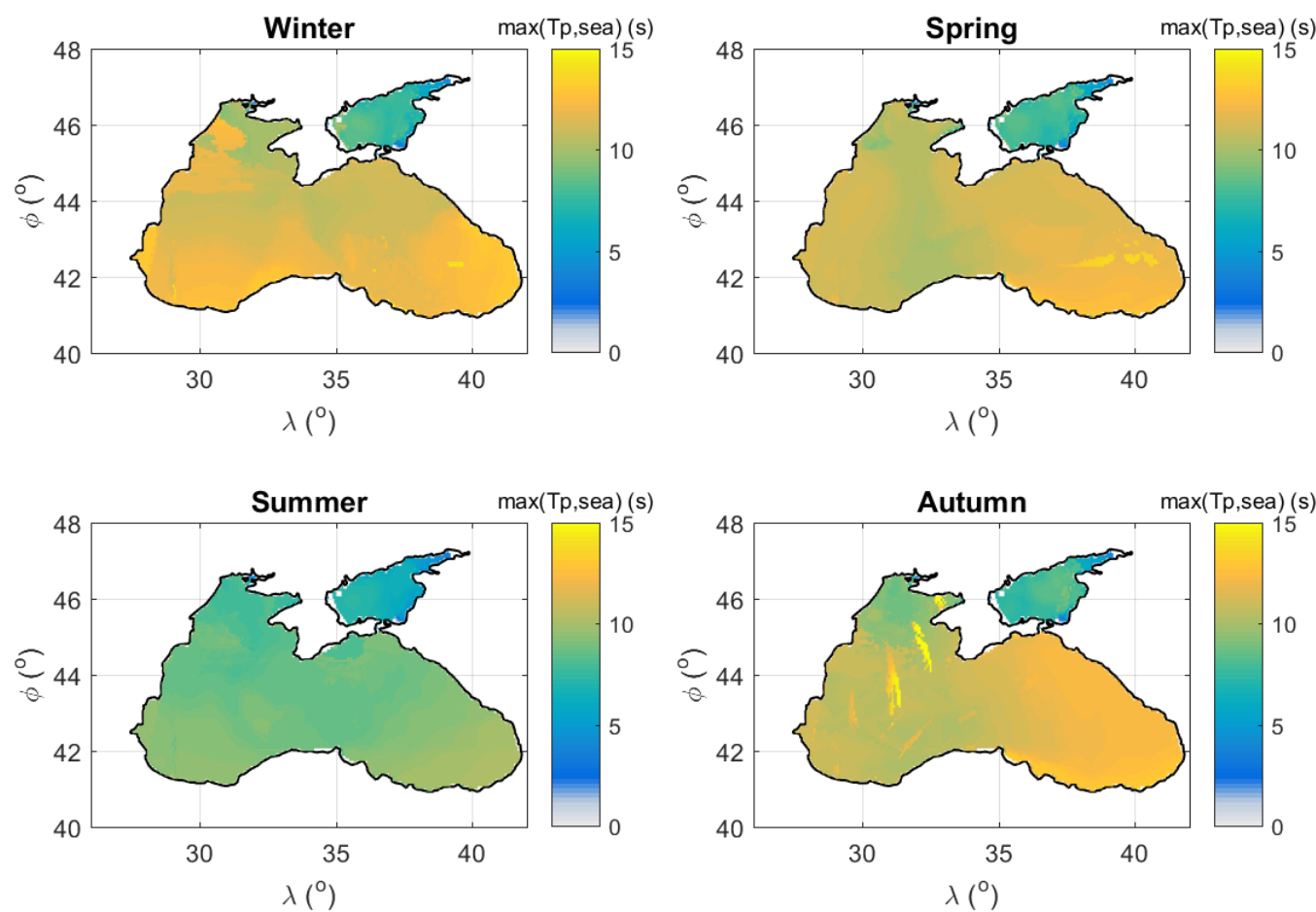

Figure 9. Spatial variation of the maximum peak period of wind seas per season based on hindcast results from 1979-2009.
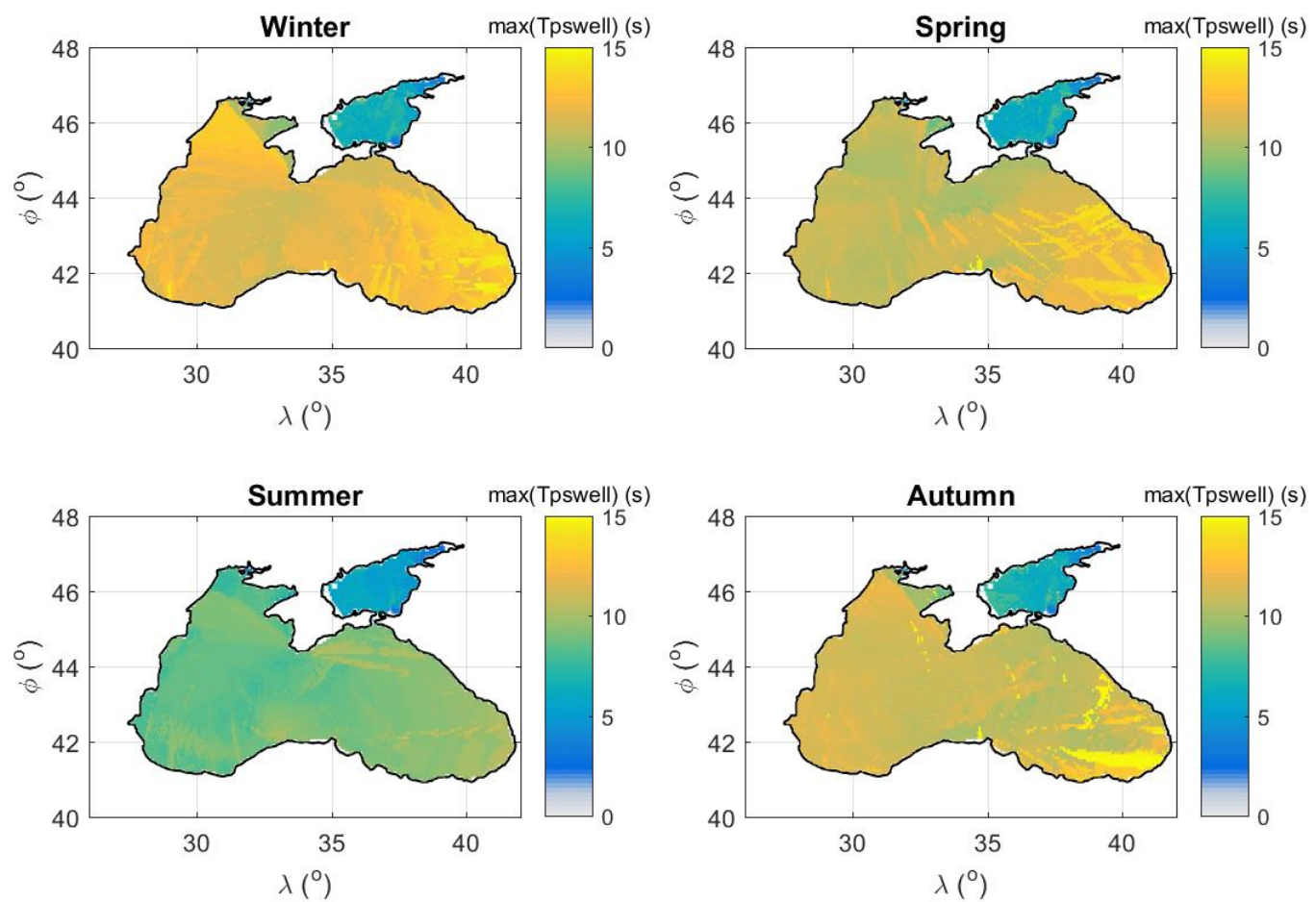

Figure 10. Spatial variation of the maximum peak period of swell waves per season based on hindcast results from 1979-2009. 

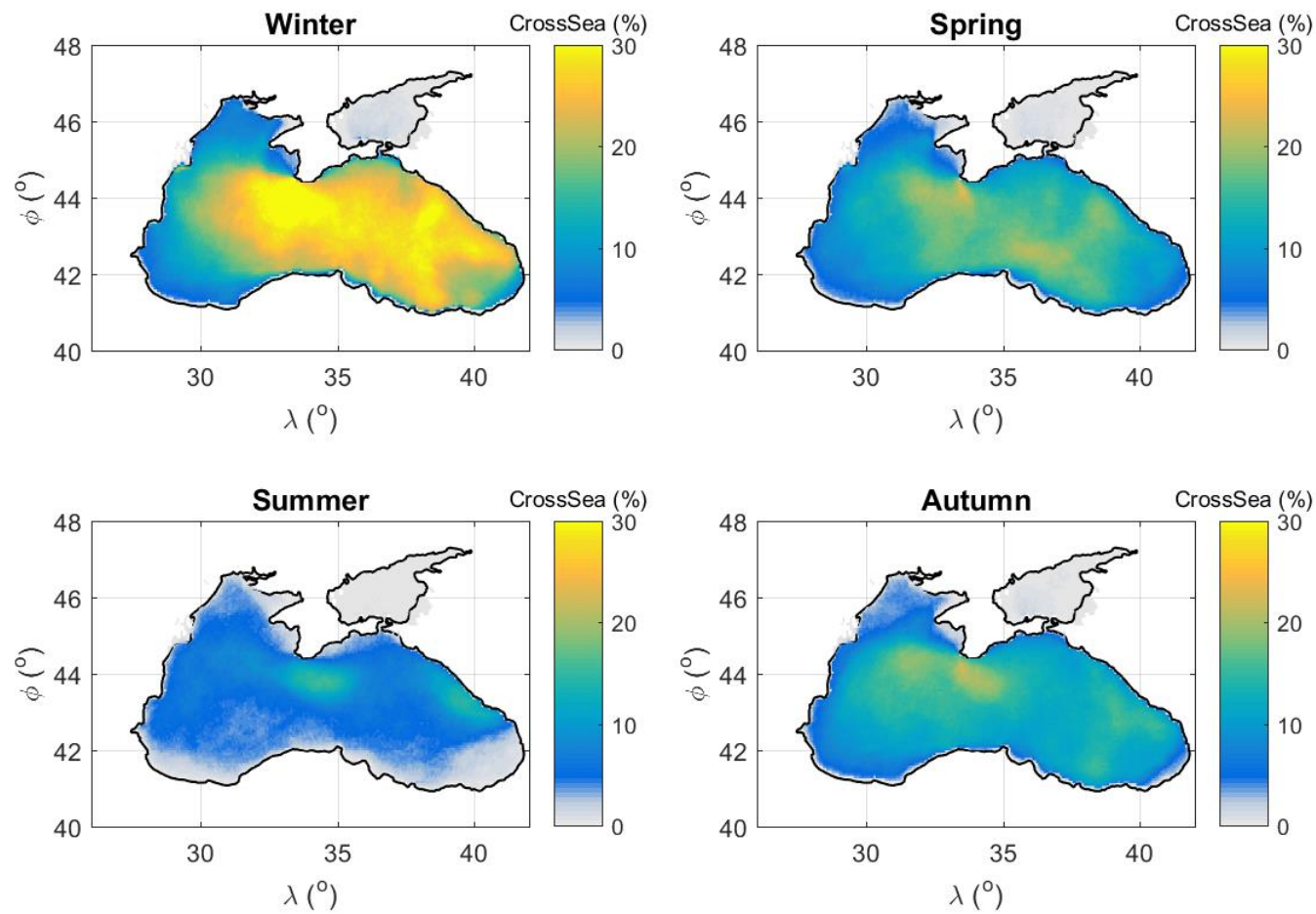

Figure 11. Spatial variation of the seasonal percentage of occurrence of dangerous cross seas based on SWAN hindcast results from 1979-2009.
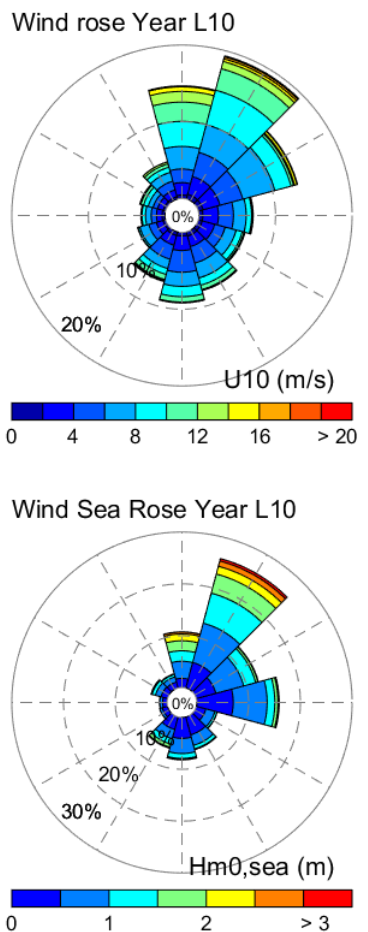
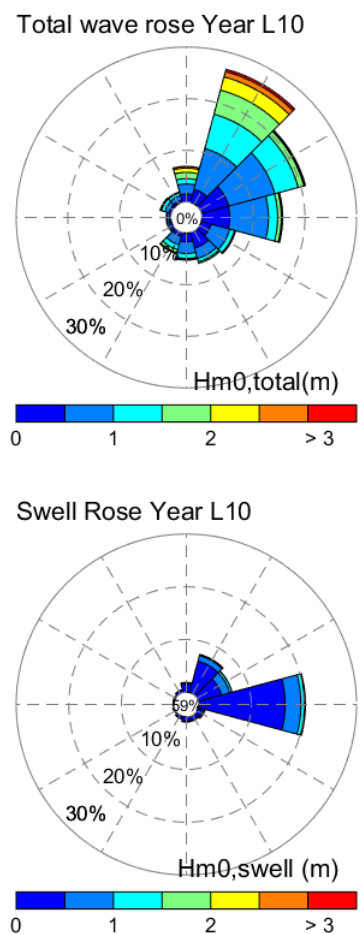

Figure 12. Annual roses for wind speed (upper left panel), total wave height (upper right panel), wind sea wave height (lower left panel) and swell wave height (lower right panel) for location 10 in the western part of the Black Sea based on SWAN hindcast results from 1979-2009. 

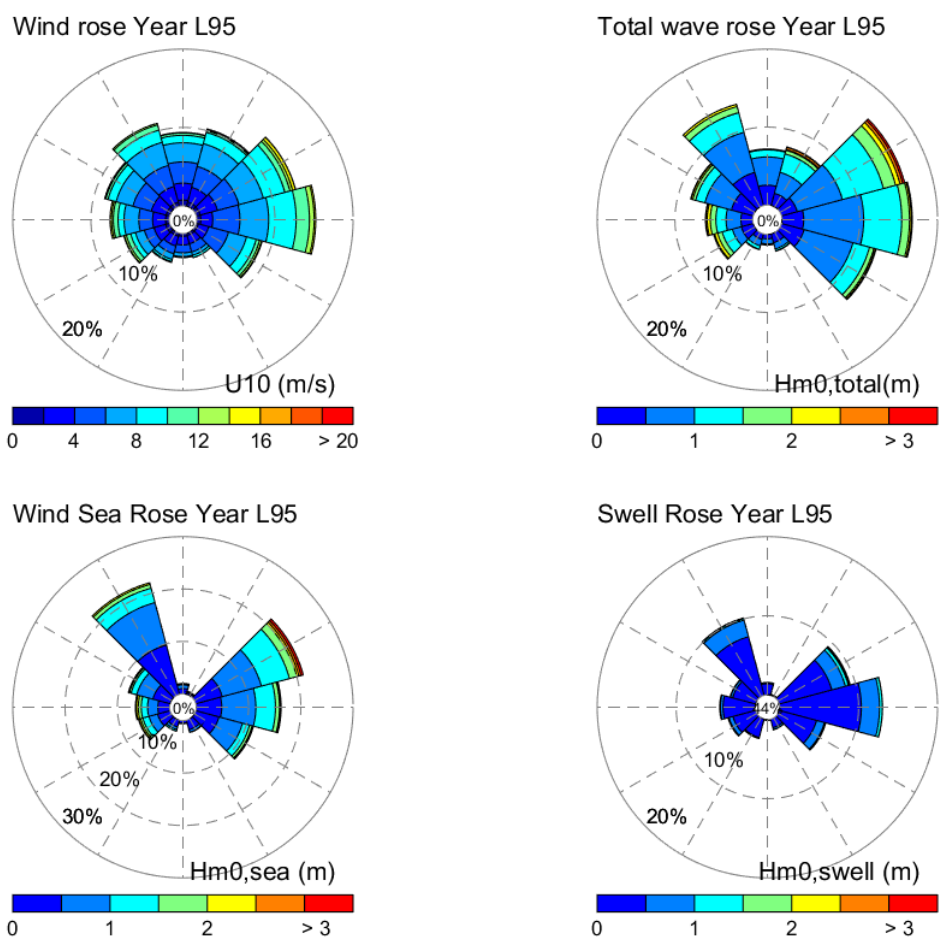

Figure 13. Annual roses for wind speed (upper left panel), total wave height (upper right panel), wind sea wave height (lower left panel) and swell wave height (lower right panel) for location 95 in the middle of the Black Sea based on SWAN hindcast results from 1979-2009.
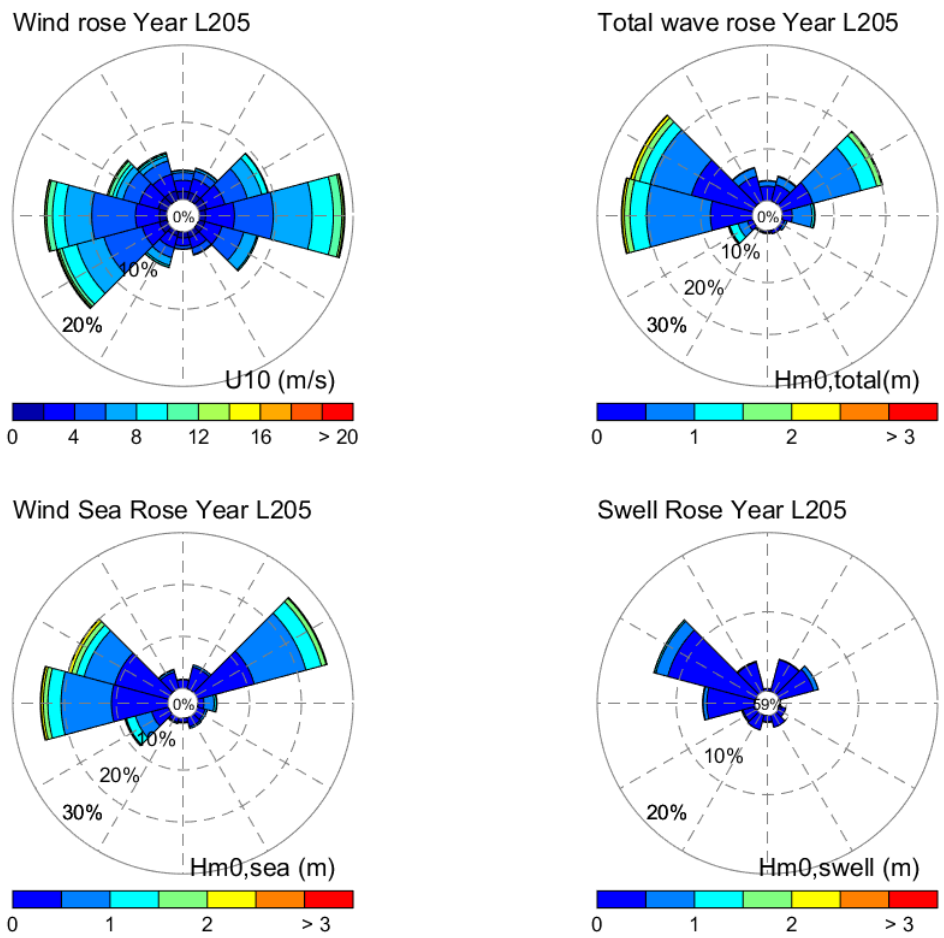

Figure 14. Annual roses for wind speed (upper left panel), total wave height (upper right panel), wind sea wave height (lower left panel) and swell wave height (lower right panel) for location 10 in the eastern part of the Black Sea based on SWAN hindcast results from 1979-2009. 


\section{DISCUSSION}

In this study an analysis was performed on the swell climate in the Black Sea. Key to this analysis is the definition of swell and the method to extract this information from 2D-wave spectra. From a physical point of view swell waves are usually considered as those waves that propagate independent of the wind, i.e. their phase velocity is higher than the local wind component in the direction of wave propagation. This definition, however, neglects the fact that individual swell wave components are part of a wave system containing many wave components affecting each other through e.g. non-linear wavewave interaction and dissipation, even when some components are still under the influence of the wind. A clear exception to this picture are regular ocean swells having travelled thousands of kilometers and showing a high amount of regularity. To identify individual wave systems the spectral partitioning technique is used under the assumption that each (significant) local peak and associated partition in the 2D-wave spectrum represents an individual wave system. Wind sea systems are those systems whose peak is under the influence of wind according to the wave age criterion. This implies that such a wave system may contain components that are not under the influence of the local wind, and conversely, swell system may contain components that are under the influence of the local wind. In the present method, the fraction of spectral area under the influence of the wind is expressed by the parameter WFRAC. This way of assigning swell waves deviates from the ECMWF method as used by Berkün (2007) where only the influence zone of wind is used as a criterion. It is noted that since 2007 the ECMWF wave products have changed significantly, see ECMWF (2015) for the most recent guide.

The spectral partitioning technique shows that even in a closed basin as the Black Sea multi-peaked wave spectra can occur composed a wind sea and one or more swell systems, whereas in the Sea of Azov significantly less multi-peaked wave spectra occurs. This difference is related to the size of the generating storm and the spatial extent of the wave basin. The larger the wave basin, the higher the probability of occurrence of swells. An important characteristic of the present spectral partitioning method is its ability to identify wave systems coming from different directions, which can be considered as an extension to the work of Yilmaz (2007). The present analysis also shows that swells can cross the entire Black Sea from its generation area in e.g. the eastern part to the other side in the West. In contrast to ocean scale basins, the effect of wave dispersion leading to relatively large swell period is limited here. Maximum swell wave periods are only slightly higher than those found in active storm areas.

The present study can be considered as an extension of the work by Berkün (2007). A qualitative comparison of the wind and wave roses shows many similarities, but also some differences. Similarities are mainly visible for the locations L10 and L205 and the nearest points used by Berkün (2007), in which dominant swell directions are both related to relatively large upwind fetches. Differences are probably due to differences in the method of swell identification, the physical and numerical settings in the wave models (ECWAM versus SWAN), and the length of the hindcast period. Further analysis are recommended to detail similarities and differences.

\section{CONCLUSIONS}

This paper shows a first application of the spectral partitioning tool in the SWAN wave model. This tool was applied to study some aspects the swell climate in the Black Sea based on hindcast results covering a period of 31 years. The results of this study lead to the following conclusions:

- Swells are common in Black Sea;

-The amount of swell energy is significant and can be up to $50 \%$, especially after the passage of a storm system;

- The spatial extent of the Black Sea is large enough for pure swells to exist (i.e. WFRAC very low);

-The highest swell wave height occurs in the winter season and in the southwestern and southeastern parts of the Black Sea. The lowest swell wave heights occur in the summer season;

- Dangerous sea states consisting of significant crossing wave systems mainly occur in the winter season;

- The maximum swell periods are slightly larger than the maximum wind sea peak periods;

- The directions from which swell waves are coming is strongly affect by upwind fetch restrictions.

-The results from this analysis are similar to those of Berkün (2007). 
The present study only showed limited information on the swell conditions in the Black Sea. More elaborate analyses will include a wider spatial coverage, a more detailed seasonal analysis and an extreme value analysis to investigate whether a maximum to swell heights exists.

\section{REFERENCES}

Akpinar, A., G.Ph. Van Vledder, M.İ. Kömürcü, and M. Özger, 2012. Evaluation of the numerical wave model (SWAN) for wave simulation in the Black Sea. Continental Shelf Research, 50-51, 80-99.

Akpınar, A., B. Bingölbali, and G.Ph. Van Vledder, 2016. Wind and wave characteristics in the Black Sea based on the SWAN model forced with the CFSR winds, Ocean Engineering, 126, 276-298.

Berkün, U., 2007. Wind and swell wave climate for the Southern part of the Black Sea. Master thesis, Middle East Technical University, Ankara, Turkey.

Booij, N., R. Ris, and L.H. Holthuijsen, 1999. A third-generation wave model for coastal regions: 1. Model description and validation. Journal of Geophysical Research, 104, 7649-7666.

Devaliere, E., J. Hanson, and R. Luettich, 2007. Evaluation of wave model performance in a North Carolina Test Bed, Proceedings 10th International Wave Hindcasting and Forecasting Workshop, North Shore, HI.

Cavaleri, L., and P. Malanotte-Rizzoli, 1981. Wind wave predictions in shallow waters: Theory and applications. Journal of Geophysical Research: Oceans, 86, 961-973.

ECMWF, 2015. User guide to ECMWF forecast products. http://www.ecmwf.int.

Hanson, J. L., and O. M. Phillips, 2001. Automated analysis of ocean surface directional wave spectra. Journal of Atmospheric Oceanic Technology, 18, 277-293.

Hasselmann, K, et al., 1973: Measurements of wind-wave growth and swell decay during the Joint North Sea Wave Project (JONSWAP), Deutch. Hydrogr. Z. Suppl. A8, 12, 95 pp.Hasselmann, S., K. Hasselmann, J.H. Allender, and T.P. Barnett, 1985. Computations and parameterizations of the nonlinear energy transfer in a gravity wave spectrum. Part II: Parameterizations of the nonlinear transfer for application in wave models. Journal of Physical Oceanography, 15, 1378-1391.

Komen, G., S. Hasselmann, and K. Hasselmann, 1984. On the existence of a fully developed wind-sea spectrum. Journal of Physical Oceanography, 14, 1271-1285.

Komen, G.J., L. Cavaleri, M. Donelan, K. Hasselmann, S. Hasselmann, and P.A.E.M. Janssen, 1994. Dynamics and modelling of ocean waves. Cambridge University Press. 554.

Portilla, J., F.J. Ocampo-Torres, and J. Monbaliu, 2009. Spectral partitioning and identification of wind sea and swell. Journal of Atmospheric and Oceanic Technology. 26, 107-122.

Portilla, J., L. Cavaleri, and G.Ph. Van Vledder, 2015. Wave spectra partitioning and long term statistical distribution. Ocean Modelling, virtual issue, 1-13.

Rogers, W.E., P.A. Hwang, and D.W. Wang, 2003. Investigation of wave growth and decay in the SWAN model: three regional-scale applications, Journal of Physical Oceanography, 33, 366-389.

Snyder, R.L., F.W. Dobson, J.A. Elliott, and R.B. Long, 1981. Array measurement of atmospheric pressure fluctuations above surface gravity waves. Journal of Fluid Mechanics, 102, 1-59.

SWAN Team. 2016. SWAN user manual. SWAN Cycle III version 41.10, Delft University of Technology, p. 123.

Van Vledder, G.Ph., and L.H. Holthuijsen, 1993. The directional response of wind waves to turning wind. Journal of Physical Oceanography, 23, 177-192.

Van Vledder, G.Ph, and A. Akpinar, 2015. Wave model predictions Black Sea: Sensitivity to wind fields. Applied Ocean Research, 53, 161-178.

Wavewatch III manual, 2009. User manual and system documentation of WAVEWATCH III TM version 3.14, Section on spectral partitioning.

Yilmaz, N. 2007. Spectral characteristics of wind waves in the Eastern Black Sea. Ph.D. thesis, Middle East Technical University, Ankara, Turkey.

Young. I.R., and G.Ph. van Vledder, 1993. The central role on non-linear four-wave interactions in wave evolution. Proc. Phil. Trans. Roy. Soc. Lond., A, 342, 505-524. 